ETHICAL AND WORLD-VIEW PHILOSOPHY 


\title{
Editors
}

Rudolf a. Makkreel and Frithjof Rodi

\author{
Advisory Board \\ KARLFRIED GRÜNDER \\ ULRICH HERRMANN \\ DAVID HOY \\ B. E. JENSEN \\ HELMUT JOHACH \\ P. D. JUHL \\ OTTO PÖGGELER \\ HANS PETER RICKMAN \\ PAUL RICOEUR \\ THOMAS SEEBOHM
}




\section{WILHELM DILTHEY}

SELECTED WORKS • VOLUME VI

\section{Ethical \\ and World-View \\ Philosophy}

EDITED, WITH AN INTRODUCTION, BY

Rudolf A. Makkreel

AND

FRITHJOF Rodi

PRINCETON U N I VERSITY PRES S

PRINCETON AND OXFORD 
Copyright (C) 2019 by Princeton University Press

Published by Princeton University Press

41 William Street, Princeton, New Jersey 08540

6 Oxford Street, Woodstock, Oxfordshire OX20 1TR

press.princeton.edu

All Rights Reserved

LCCN 2019936030

ISBN 9780691195575

British Library Cataloging-in-Publication Data is available

Editorial: Rob Tempio, Matt Rohal

Production Editorial: Debbie Tegarden

Text Design: Pam Schnitter

Jacket/Cover Design: Pam Schnitter

Jacket Credit: Frank Mahood

Production: Jacquie Poirier

Publicity: Alyssa Sanford

Copyeditor: Jay Boggis

This book has been composed in Sabon LT Std

Printed on acid-free paper. $\infty$

Printed in the United States of America

$\begin{array}{llllllllll}1 & 3 & 5 & 7 & 9 & 10 & 8 & 6 & 4 & 2\end{array}$ 